\title{
Mythopoeia und Wissenschaft in De hortis Hesperidum von Giovanni Gioviano Pontano
}

Giovanni Pontanos De hortis Hesperidum, ein Lehrgedicht in zwei Büchern von insgesamt ca. 1200 Hexametern, ${ }^{1}$ erhebt in der zeitgenössischen Literatur zu Recht den Anspruch auf absolute Originalität hinsichtlich Form und Inhalt sowie der Kombination von wissenschaftlich-botanischem Thema und mythologischer Komponente. Das Neuartige besteht vor allem in der Wahl des Themas, nämlich den Zitrusgärten und insbesondere ihrer Pflege, wobei das botanische Regelwerk von einer musiven Konstruktion verschiedenster Materialien gestützt wird, welche zudem die ursprüngliche Sage des Adonis, die Ovid in etwas mehr als 200 Hexametern erzählt (Met., 10,503 ff.), erweitern und erneuern.

Der Dichter entwirft Ursprungsmythen, die in einer ,persönlichen' Geographie verortet, doch überaus innovativ ${ }^{2}$ und anspielungsreich ${ }^{3}$ sind. So schlägt Pontano in Anlehnung an eine Reihe von klassischen Quellen in diesem den (goldenen) Äpfeln der Hesperiden geweihten botanischen Lehrgedicht eine Identifikation von Zitrusfrüchten ${ }^{4}$ mit den Äpfeln der Hesperiden vor, wobei er

1 De hortis Hesperidum wurde erstmals 1505 durch Aldo Manuzio publiziert (Ioannis Ioviani Pontani: Opera. Urania, sive de stellis libri quinque. Meteororum liber unus. De hortis Hesperidum libri duo. Lepidina sive pastorales, pompae septem. Item Meliseus, Maeon Acon. Hendecasyllaborum libri duo. Tumulorum liber unus. Naeniae duodecim. Epigrammata duodecim. Venetiis, in aedibus Aldi Romani, mense augusto 1505). Mein Text bezieht sich auf die moderne Ausgabe Ioannis Ioviani Pontani: Carmina. Hg. von Benedetto Soldati. Bd. 1. Florenz 1902, S. 229-261. Die Übersetzungen stammen, wenn nicht anders angegeben, von der Autorin.

2 Dazu Donatella Coppini: Le Metamorfosi del Pontano. In: Le Metamorfosi di Ovidio tra Medioevo e Rinascimento. Hg. von Gian Mario Anselmi, Marta Guerra. Bologna 2006, S. 75-108.

3 Giuseppe Germano: Giovanni Pontano e la costituzione di una nuova Grecia nella rappresentazione letteraria del Regno Aragonese di Napoli. In: Spolia. Journal of Medieval Studies 1 (2015), S. 36-81.

4 Die heute mit dem wissenschaftlichen Namen ,Hesperidium‘ versehenen Früchte nennt Pontano zum ersten Mal unter der Bezeichnung acrumen in Hesp. II 4; Orangen, d. h. die süße Art der Zitrusfrüchte, werden vom Dichter unter der Bezeichnung arangaeae silvae zum ersten Mal in Hesp. I 592 genannt und aufgrund der Para-Etymologie (arangio/aragonesi) als Anspielung für das Haus Aragon verwendet, s. u.

Ich danke Barbara Pfister für die Übersetzung aus dem Italienischen. 
eine nicht überlieferte mythographische Variante einbaut, welche die mala Hesperidum aus dem Körper des vom Keiler getöteten Adonis entstehen lässt. Das Aition birgt in sich eine Vielzahl von Anspielungen, vor allem auf die Fülle der Zitrusfrüchte im Königreich von Neapel und die Rolle der Orange als Symbol der Herrscherdynastie der Trastámara von Aragon. ${ }^{5}$ Die durch den Willen der Venus aus dem Körper des Adonis entstandene Pflanze wird - gerade deshalb vom Dichter als unvergängliches und zur Ewigkeit bestimmtes Gewächs zelebriert (De hortis Hesperidum, I 527-531):

Est citrio aeternum genus, immortalis origo, Et species aeterna quidem. Stirps citria longum Ipsa manet secla exsuperans, et iungere seclis Secla parans, trunco extincto mox surgit et alter, Inde alter victrixque diu sua robora servat.

Die Zitronatzitrone ist von ewiger Art, ihr Ursprung unsterblich, ihre Gattung bestimmt zur Ewigkeit. Der Baum der Zitronatzitrone lebt lange, die Jahrhunderte überdauernd und aus seinem abgestorbenen Stamm entsteht, bereit, Jahrhunderte um Jahrhunderte zu vereinen, unvermittelt ein anderer und hierauf noch einer, sodass er siegreich auf lange Zeit seine Kraft bewahrt.

Pontano, der unbestrittene Meister des neapolitanischen Humanismus, bekräftigte stolz, dass sein Lied keine Vorläufer habe (De hortis Hesperidum, I 125-127):

Nunc, age, qui cultus citriis, qua certa serendi

Tempora quaeque illis regio magis apta ferendis

Expediam nullique loquar memorata priorum. ${ }^{6}$

Nun auf! Welche Anbauarten sich für die Zitronatzitronen eignen, welches die Zeiten der Aussaat sind, welches die geeignetsten Orte sie zu züchten werde ich erklären und erzählen, wovon keiner vor mir gekündet hat.

Der Mythos von der Liebe der Venus zu Adonis bildet den Kern des Werks ungeachtet dessen vorwiegend botanischen Inhalts. Pontano baut das Gedicht

5 Vgl. dazu Antonietta Iacono: Il De hortis Hesperidum di Giovanni Pontano tra innovazioni umanistiche e tradizione classica. In: Spolia. Journal of Medieval Studies 1 (2015), S. 188-237.

6 Pontano zelebriert im Verlauf des Gedichtes die Originalität seines Werkes, indem er die Seltenheit der Pflanze unterstreicht, rarum decus hortis (De hortis Hesperidum, II 261), die auf das Gedicht zurückstrahlt, deren Gegenstand sie ist, womit dieses zum rarus labor wird (De hortis Hesperidum, II 218-219): „tertia iam superat limonis cura colendae / et rarus labor et coepti meta ultima nostri“. Auch im Dialog Aegidius bekräftigte der Humanist noch einmal die Originalität dieses Werkes: Giovanni Pontano: Dialoghi, a cura di Carmelo Previtera. Florenz 1943, S. 261. 
auf einer aus der klassischen Mythologie bekannten Metamorphose auf, jedoch mit signifikanten Unterschieden: während Ovid (Met., 10,735-739) den jungen Liebhaber der Venus in eine Anemone verwandelt und Bion von Smyrna in eine purpurfarbene Rose (Epitafio, V. 66), wird Adonis hier zu einem Zitronatzitronenbaum. Im ersten Buch, das einem ausführlichen Regelwerk zur Kultivierung der Zitronatzitrone vorangestellt ist, erklären eine Reihe von neuen Aitia, wie diese Pflanze nach Italien und im Speziellen an die Küsten und in die Gärten Neapels gelangte: sie erzählen vom Tod des Adonis und seiner Metamorphose in einen Zitronatzitronenbaum; dass die Frucht von Herkules der Nymphe Ormiale anvertraut wurde (I 102-124), die in Pontanos mit gelehrten Verweisen gekoppelter Neuschöpfung wiederum einen bestimmten Ort darstellt, Formia, das antike Hormiai; ${ }^{7}$ dass die Pflanze durch das Aufbegehren Junos, die sich für den Raub der Äpfel aus dem ihrem Schutz unterstellten Garten der Hesperiden rächen wollte, e Medorum divite sylva den Nachfahren des Aeneas überbracht wurde (I 168-188); das Buch endet unerwartet und faszinierend mit einem Zauber der Parzen (I 526-607), einem großartigen Ursprungsmythos zur Erklärung, weshalb der Zitronatzitronenbaum eine ewige Pflanze ist. Nach der komplexen mythologisch-ätiologischen Einleitung mit der Metamorphose des Adonis und der Erzählung der Ankunft der Zitronatzitrone in Italien beginnt die Entwicklung eines ausführlichen und detailgenauen Regelsystems zum Anbau der Zitrusfrüchte, das folgende Themen behandelt: die geeigneten (125-146) und nicht geeigneten Orte zum Anbau (147-167); die zur Pflanzung geeignete Zeit (189-199); die aufgrund von Kälte nicht geeigneten Regionen (200-231); die Tiefe der Pflanzlöcher (232-240); der zur Pflanzung geeignete Boden (241-253); der Beschnitt der Pflanzen (254-259); die Beschaffung von Saatgut (260-280); die Art der Aussaat (281-310); saure und süße Zitrusfrüchte (336-373); wie man besonders große Früchte erhält (374-379); wie man ganzjährig Früchte erhält (380-386); die Pflege der Zitrusgärten (387-451); kein Beschnitt der Pflanze nach der kalten Jahreszeit (452-464); die Ordnung und Verteilung der Bäume im Garten (465-494); die Planung eines Gartens (495-525).

Auch die erzählerische Entwicklung des zweiten Buches ist bestimmt von der Schichtung neu erfundener oder auf dem Kern antiker Mythen erneuerter Aitia und deren Verflechtung mit lehrhaften Inhalten. In dieser symmetrischen Struktur wechseln sich die ätiologischen mit den botanischen Abschnitten ab, wobei erstere wiederum den erzählerischen Unterbau und die fabulös-phantastische

7 Siehe Plin. Nat., 3,59: „Formiae, Hormiae prius dictae olim, antiqua sedes Lestrigonum“; und Strab. 5,3,6. 
Legitimation liefern. Nach einer langen und komplexen Einleitung mit erneutem Anruf der kampanischen Nymphen und nochmaliger Widmung an Gonzaga (1-51), entwickelt Pontano eine Reihe von Regeln zu den Zitrusfrüchten und deren Anbau (52-179); erklärt den Unterschied zwischen citrius (Gurke) und citrus (Zitronatzitrone: 180-217); fokussiert sich auf den Anbau der Zitronen (218-308); erklärt die Art und Weise der Veredlung (309-384); unterscheidet zwischen süßen und sauren Zitrusfrüchten (432-499); geht schließlich auf die Verwendung der Früchte ein (500-523), um dann zur langen Schlussstrophe überzuleiten (524-581). Im Abschnitt, der den Zitronen gewidmet ist, gibt es weitere Teilabschnitte: zunächst die genaue Bestimmung von drei Arten von Zitronen (246-252); weiter ein Rezept zur Herstellung einer Salbe aus dem Saft von sauren Zitronen, die der Haut der Frauen wieder Glanz schenkt (253-256); schließlich die Etablierung von Mergellina als Kultstätte der Zitrusfrüchte - Mergellina, das Sannazaros Exil in Frankreich beweint (289-308). Der mythologische Exkurs von der Liebe Alkyones zu Neptun, und wie es der Nymphe dank des Duftes der Orangenblüten gelingt, den Meeresgott $\mathrm{zu}$ betören (196-217), fügt sich nicht zufällig in einen Abschnitt zum Anbau der Zitronatzitrone, in dem Pontano auch den Unterschied zwischen citrius und citrus erklärt (180-217). Eine Reihe von mythischen und ätiologischen Bildern findet sich auch im Abschnitt zum Anbau der Zitronen (218-308): so die Apostrophe, in welcher der kleine Amor seine in der fons Nisaeius badende Mutter Venus aufruft, mit ihm nach Amalfi zu fliegen (230-239); oder auch die Entscheidung der Grazien, den Jungvermählten als Brautgabe Orangenblüten darzureichen (240-268). Und schließlich der eindeutige Bezug des Abschnitts zu den Veredelungstechniken (309-406) auf den Lobpreis menschlicher Arbeit und Mühen - Laudes Industriae humanae - als Streben nach Erlösung und Fortschritt im darauffolgenden dichterischen Abschnitt (407-431).

Pontanos Erfindung des Adonis-Mythos stellt nicht die einzige Neuheit dar, um die herum das Gedicht entwickelt wird: ein wichtiges Verbindungsglied ist die Gleichsetzung der Zitrusfrucht-Gärten mit den Gärten der Hesperiden. Diese ist zweifellos Ausgangspunkt des Mythos und des Werkes. Gemäß dem heutigen Stand meiner Forschung war Pontano der Hauptverfechter dieser Identifikation der mythologischen Gärten mit einem konkreten geographischen Ort, nämlich Kampanien, das aufgrund dessen sprichwörtlicher Fruchtbarkeit und der Produktivität seines Territoriums zum Mythos der Hesperiden und dem Reichtum ihrer Gärten passte. Diese Adaptation wird von klassischen Quellen unterstützt, wie zum Beispiel Athenaios (Deipnosofisti, III 83), der die Zitronatzitrone als

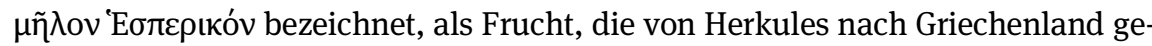

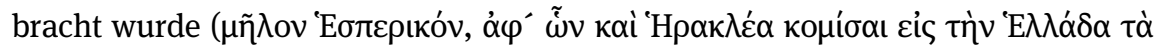

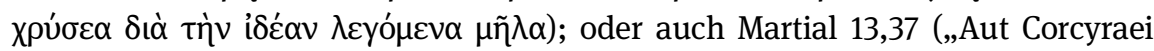


sunt haec de frondibus horti, / Aut haec Massyli poma draconis erant“), der die Zitronatzitronen als poma draconis identifiziert und damit auf den Wächter des Gartens der Hesperiden anspielt. ${ }^{8}$

Neben den eindeutigen kosmologischen Bezügen des Adonis-Mythos, die im Detail von Macrobius in den Saturnalia $(21,1)$ erklärt werden (eine Quelle, die Pontano aufgrund seiner astronomisch-astrologischen Interessen studierte) ${ }^{9}$, war der Humanist sicher von der symbolischen Vielfalt des Mythos und der Figur des Adonis fasziniert: eine aus einem Inzest geborene Kreatur, Sohn der Myrrha und damit Personifikation jenes Harzes, das der Einbalsamierung diente und der ewigen Reise der Toten ins Jenseits; auch Symbol der Teilung des Lebens in Jahreszeiten; das Objekt einer unglücklichen, leidenschaftlichen Liebe; Protagonist eines Mythos, der den verfrühten Tod eines Jünglings vorsah; nicht zuletzt verbunden mit den Festen der Adonia, die in der gesamten Antike, in der semitisch wie griechisch-römischen Welt verbreitet waren und von denen Pontano vermutlich durch die Lektüre von ihm geschätzter Autoren Kenntnis hatte. ${ }^{10}$ Die Gründe für die Wahl des Adonis als Protagonisten eines Gedichtes, das die Wesenszüge eines Epyllions trägt, sind also nicht zuerst in der Wiederentdeckung Theokrits und der griechischen Bukolik im Europa des fünfzehnten Jahrhunderts zu suchen, die die Adonis-Figur in neues Licht rückte und wieder einziehen ließ in alle Literaturgattungen, ${ }^{11}$ sondern vielmehr im Bestreben des Humanisten, den antiken und vergänglichen Gärten der Adonia $^{12}$ die neuen und unsterblichen Gärten des Adonis mit ihren Zitronatzitronen (anspielungsreiches Symbol) sowohl literarisch als auch konkret entgegenzusetzen.

8 Die Gleichsetzung der Zitronatzitronen mit den mala Hesperidum erlaubt es Pontano, seine Neuschöpfung an die Taten des Herkules zu binden, eines Helden, für den er auch aus persönlichen Motiven eine besondere Vorliebe nährte. Siehe hierzu Iacono (Anm. 5), S. 197-198.

9 Wie zum Beispiel die beeindruckende Verwendung des Autors durch Pontano in dessen Gedicht Urania zeigt. Siehe hierzu Dennis Weh (Hg.): Giovanni Pontanos Urania Buch 1. Wiesbaden 2017, passim.

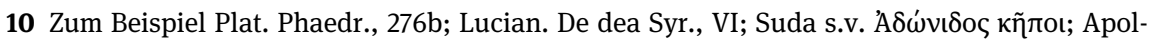
lod. Bibl., 3,14,4.; Plin. Nat., 19,19,49.

11 Zur breiten Rezeption von Theokrit im Bereich der Schola Neapolitana siehe Carlo Vecce: Un codice di Teocrito posseduto da Sannazaro. In: L'antiche e le moderne carte. Studi in memoria di Giuseppe Billanovich. Hg. von Antonio Manfredi, Carlo Maria Monti. Padua 2007, S. 596-616 sowie Carmela Vera Tufano: Il Polifemo del Pontano. Riscritture teocritee nella Lyra e nell'Antonius. In: Bollettino di Studi Latini 40/1 (2010), S. 22-43.

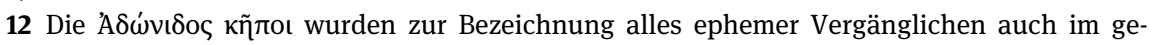
flügelten Sinn eingesetzt: Stefano Amendola: I giardini di Adone. Plut. Ser. Num. 560 B-C ed Erasm. Adag. I 1, 4. In: Paroimiakos. Il proverbio in Grecia e a Roma. Hg. von Emanuele Lelli. Bd. 3. Pisa, Rom 2010 (=Philologia Antiqua 4 [2011]), S. 123-132. 
Pontano liebte es zeitlebens, seinen Hang zum Landbau auszustellen und sich als Eigentümer einer Villa nebst Gärten in Antignano auf dem Hügel des Vomero in Neapel wie auch eines Hofes im Gebiet von Paturchio, in dem man Vergils Villa vermutete, zu präsentieren. ${ }^{13}$ So sieht man den Dichter auch in $D e$ hortis Hesperidum zum Beispiel bei der Pflege seines Gemüsegartens an der Seite seiner Ehefrau (I 195-198), wobei die Erinnerung an die gemeinsame Pflege ihres Zitrusgartens die Gelegenheit bietet, der Trauer um ihren Tod Ausdruck zu verleihen (I 318-335). Überdies kodifiziert der Humanist in den 1490er Jahren den Garten als Ort der Zerstreuung und Schauplatz von Festen, ganz in Übereinstimmung mit der hedonistischen Ethik des aragonischen Hofes von Neapel. In De splendore, 1498 publiziert zusammen mit anderen kleinen Traktaten (De liberalitate, De beneficentia, De magnificentia, De conviventia), die als Kapitel einer den Tugenden des Herrschers und Edelmannes gewidmeten Sammlung konzipiert sind, bezeichnet Pontano die Zitronatzitrone als ornamentale Pflanze, die sich aufgrund ihrer prächtigen Üppigkeit besonders gut für die fürstlichen Gärten eignet:

Erunt autem horti hi ex peregrinis et egregiis arbusculis artificiose decenterque dispositi. In quibus e myrto, buxo, citrio, rore marino topiarum opus potissimum commendatur.

Diese Gärten werden mit exotischen und seltenen Pflanzen kunstvoll und mit der gebotenen Sorgfalt angeordnet sein. Besonders erwünscht ist in diesen die achtsame und genaue Anordnung von Myrten, Buchsbäumen, Zitronatzitronen und Rosmarin. ${ }^{14}$

Pontanos Lehrsamkeit und Kompetenz in Sachen Botanik ist nicht verwunderlich. Der Humanist hatte eine besondere Vorliebe für die Wissenschaftskultur, wie schon das frühe Debüt im Genre des Lehrgedichts mit den Werken Meteororum liber und Urania ${ }^{15}$ und ebenso seine wissenschaftlich-astronomische Produktion, die durch das Studium der spezifischen griechisch-lateinischen Literatur

13 Erasmo Percopo: Vita di Giovanni Pontano, a cura di Michele Manfredi, Napoli 1938, S. 42. 14 Giovanni Pontano: I libri delle virtù sociali, a cura di Francesco Tateo. Roma 1999, S. 241244. Es handelte sich bereits um einen etablierten Trend, wie es im Fall der Villa Poggio Reale zu sehen ist, deren Bau von Alfons, dem Herzog von Kalabrien und Sohn von Ferrante I. angeordnet wurde. Der Garten der 1488 eingeweihten Villa war mit Zitrusfrüchten bepflanzt. Hierzu Paola Modesti: Le delizie ritrovate. Poggioreale e la villa del Rinascimento nella Napoli aragonese. Firenze 2014, S. 29-61, und S. 46 Tabelle 1.

15 Die beiden Gedichte wurden erstmals von Aldo Manuzio in der bereits zitierten Ausgabe von 1505 (Anm. 1) gedruckt. Siehe hierzu Benedetto Soldati: La poesia astrologica nel Quattrocento. Ricerche e Studi. Firenze 1906; Mauro de Nichilo: I poemi astrologici di Giovanni Pontano: storia del testo, con un saggio di edizione critica del Meteororum liber. Bari 1975; Weh (Anm. 9). 
unterstützt wurde, zeigt. Im Bereich der Botanik gab es natürlich, trotz der vom Autor wiederholten Beteuerung der absoluten Originalität und des Primates, bereits Vorläufer in der antiken Literatur: das zweite Buch der Georgica von Vergil, das zehnte Buch der De re rustica von Columella; ${ }^{16}$ das kleine Gedicht zur Veredelung der Pflanzen von Palladio, das wiederum als 15. Buch der De re rustica überliefert ist. Im Bereich der umfangreichen lateinischen Prosaliteratur ist an die Werke (oder was davon übrig ist) von Varro, Cato dem Älteren, die Naturalis Historia von Plinius dem Älteren (insbesondere die der Botanik gewidmeten Bücher XII-XIII-XIV-XVI-XVII-XIX-XX-XXI-XXII-XXIII) zu erinnern. Aus der griechischen Fachliteratur verdienen die botanischen Abhandlungen von Theophrast (Historia Plantarum und De causis plantarum) ${ }^{17}$ und die Geoponica, eine regelrechte Enzyklopädie des Pflanzenbaus von außerordentlicher Popularität, ${ }^{18}$ Erwähnung.

In diesem Panorama illustrer Vorläufer ist auch auf die Tradition der Schola medica Salernitana und ihre außergewöhnliche Kompetenz auf dem Feld der Botanik und Pharmazeutik zu verweisen. Pontano nährte für die schola eine

16 Columella, wiederentdeckt von Poggio Bracciolini, galt wohl als wertvoller Autor, der sowohl auf dem Feld der Exegese als auch der literarischen Nachbildung besonderer Aufmerksamkeit würdig war. Einer der ersten Kommentare zum zehnten Buch der De cultu hortorum ist jener von Pomponio Leto, erstmals im Jahr 1472 in anonymer Form publiziert $(=\mathrm{H}$ 5497; IGI 3067): Giancarlo Abbamonte: Intuizioni esegetiche nel suo commento alle Georgiche e all'Eneide di Virgilio. In: Esegesi dimenticate di autori classici. Hg. von Carlo Santini, Fabio Stok. Pisa 2008, S. 135-210, hier S. 154-157. Die Verbreitung von Columella in Neapel ist überdies dokumentiert durch eine Bilderhandschrift im Bestand der aragonischen Bibliothek (heute: Valencia, Biblioteca Universitaria, ms. 740): Tammaro de Marinis (La biblioteca dei re d'Aragona. Bd. 2. Milano 1947, S. 51).

17 Man erinnere sich, dass es von Theophrasts De causis Plantarum eine lateinische Übersetzung von Teodoro Gaza gab, 1483 gedruckt in Treviso, die Pontano mit aller Wahrscheinlichkeit bekannt war: Daniela Gionta: Il codice di dedica del Teofrasto latino di Teodoro Gaza. In: Studi Medivali e Umanistici 2 (2004), S. 167-214. Im Übrigen wird ein „Theophrasto, in greco, ad stampa“ im Inventar der Bibliothek Pontanos gelistet, die seine Tochter Eugenia der Bibliothek von San Domenico Maggiore in Neapel vermachte: Michele Rinaldi: Per un nuovo inventario della Biblioteca di Giovanni Pontano. In: Studi Medievali e Umanistici 5-6 (2007-2008), S. 163-201, hier S. 182.

18 Ein Kodex der Sammlung existierte in der Privatbibliothek von Lorenzo de' Medici (=Laur. Plut. 59.32) bereits im Jahr 1491. Zu dieser Handschrift siehe Sebastiano Gentile: I codici greci della biblioteca medicea privata. In: I luoghi della memoria scritta. Manoscritti, incunaboli, libri a stampa di Biblioteche statali italiane. Hg. von Guglielmo Cavallo. Rom 1994, S. 115-121, hier S. 117. 
besondere Bewunderung wie die wiederholten Zitate in De luna ${ }^{19}$ und im archäologisch-antiquarischen Zusatz von De bello Neapolitano ${ }^{20}$ zeigen.

Dass das Interesse an der salernitanischen Wissenstradition von den am aragonischen Hof aktiven Intellektuellen geteilt wurde, findet einen bedeutenden Beleg im Druck des Opus Pandectarum Medicinae (einer Sammlung von Mineralstoffen mit Angabe ihrer pharmazeutischen Qualitäten) von Matteo Silvatico, einem der bekanntesten Vertreter der Schola Salernitana, der im Jahr 1474 von Angelo Catone, dem Arzt Ferrantes I. von Aragon und Dozenten am studium Neapels $^{21}$ veranlasst wurde. Catone ließ die Pandette nach dem Wiederauffinden einer Kopie in der königlichen Bibliothek unter dem Titel Liber cibalis et medicinalis pandectarum Mathei Silvatici. Angelus Cato curavit ${ }^{22}$ drucken. Im Vorwort erläutert er Anlass und Beweggründe für den Druck des in überaus schlechtem Zustand aufgefundenen Textes:

Quod cum fuerit per ea tempora magno studio in regia bibliotheca asservatum, nequaquam est ita vulgatum ut transcribi a multis commode potuerit. [...] Sed quoniam indignum est ut auctoris eius nomen excellensque ingenium atque industria penitus ab hominum memoria deleta videatur, ut etiam pateat fructus quem eo duce possunt adipisci mortales, inventum fere nuper et ad nostrum seculum revocatum, opus magna tamen ex parte depravatum emendare et dare in lucem atque in communem usum nuper institui. ${ }^{23}$

Das Werk ist lange Zeit mit großer Sorgfalt in der königlichen Bibliothek verwahrt worden, jedoch war es nie so verbreitet, dass es leicht hätte abgeschrieben werden können. [...] Da

19 Michele Rinaldi: Il De luna liber di Giovanni Pontano, edito con traduzione e commento secondo il testo dell'editio princeps napoletana del 1512. In: Atti della giornata di studi per il V centenario della morte di Giovanni Pontano. Hg. von Antonio Garzya. Neapel 2004, S. 72-119, hier S. 101.

20 Antonietta Iacono: Geografia e storia nell'Appendice archeologico-antiquaria del VI libro del De bello Neapolitano di Giovanni Gioviano Pontano. In: Forme e modi delle Lingue e dei testi tecnici antichi. Hg. von Raffaele Grisolia, Giuseppina Matino. Neapel 2012, S. 160-214, hier S. 194-195.

$21 \mathrm{Zu}$ Angelo Catone siehe Bruno Figliuolo: La cultura a Napoli nel secondo Quattrocento. Udine 1997, S. 279-407.

22 Corinna Bottiglieri: Le Pandette di Matteo Silvatico dalla corte di Roberto d'Angiò alla prima edizione a stampa (Napoli 1474). In: Farmacopea antica e medioevale. Atti del Convegno Internazionale di Studi di Salerno, 30 novembre-2 dicembre 2006. Salerno 2009, S. 251-268; Corinna Bottiglieri: Appunti per un'edizione critica del Liber Pandectarum medicinae di Matteo Silvatico. In: La scuola Medica Salernitana. Gli autori e il testo. Convegno internazionale, Università degli Studi di Salerno, 3-5 novembre 2004. Hg. von Danielle Jacquart, Agostino Paravicini Bagliani. Florenz 2007, S. 31-58.

23 Ich übernehme hier die von C. Bottiglieri in Il testo e le fonti del Liber pandectarum medicinae di Matteo Silvatico. Osservazioni e rilevamenti di una ricerca in corso. In: Kentron 29 (2013) vorgeschlagene Textvariante fructus quem eo duce. 
es aber nicht rechtens wäre, wenn der Name, das hervorragende Talent, der Intellekt und die Mühe des Autors ganz aus dem Gedächtnis der Menschheit gestrichen würden, damit aber der Ertrag, dessen die Sterblichen unter seiner Führung habhaft werden können, sichtbar werde, habe ich beschlossen, nachdem ich das zu großen Teilen beschädigte Werk jüngst wiedergefunden und unserer Zeit zurückgegeben habe, es aufzuarbeiten und zu drucken und allen zum Gebrauch zur Verfügung zu stellen.

Die Edition der Pandette des Silvatico entsteht demnach innerhalb der philologischen Werkstatt der königlichen Bibliothek, ${ }^{24}$ aufgrund der spezifischen Interessen Angelo Catones, aber auch dank der am Hof allgemein verbreiteten Aufmerksamkeit für die mit einer illustren Tradition des Reiches verbundene Wissenschaftskultur, die in Funktion eines klaren Vorhabens - der Nobilitierung des Regno und seiner Geschichte - wiederbelebt wurde. Pontano zitiert also nicht zufällig die Schola Salernitana und ihre Doktoren im Verlauf der De hortis Hesperidum. Er bezeichnet sie dabei ausdrücklich als berühmte Vorläufer (II 519-23):

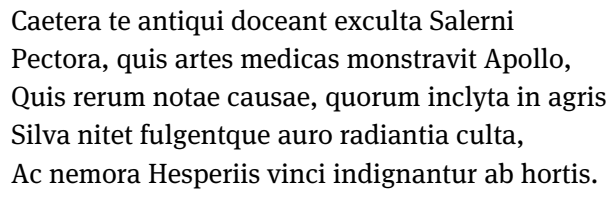

Das Übrige werden dich die gebildeten Herzen des antiken Salerno lehren, denen Apollo die medizinischen Künste zeigte, denen die Ursprünge des Kosmos bekannt sind, deren ruhmvoller Wald auf dem Lande strotzt, deren Felder von Gold erstrahlen und deren Haine sich empören, von den Gärten der Hesperiden übertroffen zu werden.

Der abschließende Vers beansprucht auffällig den Primat der Schola Pontiniana (gleichgesetzt meiner Ansicht nach mit horti Hesperii) über die Schola Salernitana. Dieser Anspruch hat mehrfache Bedeutungen (vielleicht auch in Bezug auf eine traditionelle Feindschaft zwischen salernitanischem und neapolitanischem Studium), bezeichnet hier aber wohl auch einen unterschiedlichen Ansatz in der botanischen Wissenschaft, die in der Schola Salernitana rein pharmazeutischen Zwecken diente, während die Botanik in Pontanos Lehrgedicht als universelle Wissenschaft dargestellt wird: sie erfährt Anwendung bei der Kultivierung der Zitrusfrüchte und der mit Zitrusfrüchten bestellten Gärten, trägt jedoch auch dem Kult des Schönen Rechnung, der Harmonie und der voluptas, welche der Humanist in all seinen Werken als konnotative Chiffren des Regno di Napoli feiert.

$24 \mathrm{Zu}$ den Beziehungen der königlichen Bibliothek (auch scriptorium) mit einer Reihe von in Neapel tätigen Druckern siehe Gabriella Albanese: Tra Napoli e Roma. Lo scriptorium e la biblioteca dei re d’Aragona. In: Roma nel Rinascimento. 1997, S. 73-86. 
Pontano kreiert also ein Gedicht, in dem Mythos und Wissenschaft eng miteinander verflochten sind. Aus dem Adonis-Mythos - der Liebhaber der Venus, getötet vom Eber und durch die Göttin verwandelt in einen Zitronatzitronenbaum, erfährt in dieser Form seine Bestimmung zum Schmuck fürstlicher Gärten und zum Gegenstand sublimer Dichtung - entwickeln sich eine Reihe von komplementären Mythen, die dazu dienen, spezifische Eigenschaften der Zitrusfrüchte $\mathrm{zu}$ erläutern (zum Beispiel das Vorkommen von süßen wie sauren Zitrusfrüchten), ihre Ankunft und Verbreitung in bestimmten Orten zu erklären (wie zum Beispiel die Zitronen von Amalfi) sowie ihre Verwendung zu legitimieren (wie zum Beispiel die Orangenblüten als Schmuck der Brautgemächer). Die Schichtung dieser Ursprungsmythen ist engmaschig, detailreich, sie formt das Gedicht als narratives Kontinuum sagenhafter Segmente, die sich in den wissenschaftlichen Lehrinhalt einweben - als Vorgeschichte, als Aition der Praxis von Anbau, Verwendung und Verbreitung von Zitrusfrüchten.

Einige Beispiele:

Die Verbreitung von süßen Zitrusfrüchten (äußerst selten in Italien und nur verbreitet in der Gegend von Gaeta-Formia, ${ }^{25}$ das Teil des Königreichs Neapel war) beziehungsweise sauren, nicht essbaren Früchten (allgemein verbreitet in Italien und Griechenland), ist direkt verbunden mit dem Mythos des Adonis, seinem Tod und der Rolle, die Venus der aus dem Körper ihres Geliebten entstandenen Zitruspflanze zuweist (De Hortis Hesperidum, I 335-345):

Est vero et duplex citrii genus, et quod amores Iucundos referat dulces et Adonidis ignes,

(Sic placitum Veneri) dulce hoc; quodque acre dolores

Et tristes luctus et lamentabile funus

Sorte refert, suus ut lacrimis ne desiit amaror.

Rara sed Ausonio Lamiaeque in litore foetus

Dat dulces, succosque frequens meditatur acerbos;

Contra solis ad ortum atque in Gangetide terra

Sponte sua dulcem victum ac redolentia ramis

Mella liquat, fluit eque indis liquor atticus hortis.

Doppelt ist in der Tat die Gattung der Zitronatzitrone: die erste ist süß, da sie an die Liebe und die verspielte Glut des Adonis erinnert (so wie es Venus gefiel); und die andere ist sauer, erinnert den Schmerz und das traurige Sterben, die Klage und Tränen der Totenfeier, auf dass den Tränen niemals die Bitternis fehle. Doch selten ist in Italien und an Ausoniens und Lamias Gestade die Pflanze, die süße Früchte schenkt, oft stellt sie saure

25 Ausonio Lamiaeque in litore bezeichnet die Küste von Kap Circeo, insbesondere das Gebiet von Formia, in Übereinstimmung mit den klassischen Quellen, darunter Ov. Met., 14,233. Siehe auch De hortis Hesperidum, I 582. 
Säfte bereit; im Orient dagegen und im Land des Ganges schenkt sie freiwillig süße Nahrung und träuft aus Ästen duftend süße Säfte, aus den Gärten Indiens strömt ein Likör gleich dem Honig Attikas.

Pontano lässt sich bei der Unterscheidung der Früchte, die er mit Schlüsselmomenten aus dem Mythos des Adonis, seinem verfrühten Tod und den Verfügungen der Venus zu untermauern scheint, ebenfalls von den geographischen Entdeckungen seiner Zeit inspirieren: so beschreiben die folgenden Zeilen (346-361) das Vorkommen von süßen Zitrusfrüchten in Indien an den Ufern des Ganges, womit die Umsegelung Afrikas durch Vasco da Gama bis hin zum Ganges erinnert und poetisch umformt wird: ${ }^{26}$

Nuper enim hesperio oceano Calletia pubes

Digressa, ignotosque locos et inhospita sulcans

Aequora, non solitos tractus, nova litora obivit, Et procul arentis Libyae penetravit arenas

Audax, nec notas, pontus quas circuit, urbes.

Hinc Austro approperans coeloque intenta cadenti

Sideraque adverso servans labentia mundo, Incidit obscurum gelidi Aegocerontis in orbem

Attonita et rerum novitate umbrisque locorum; Inde pedem referens Prassi convertit ad oras,

Barbaricumque fretum exsuperans Rhaptique procellas,

Tandem gemmiferos Indi defertur ad amnes

Litoraque e citriis semper fragrantia silvis,

E quibus hyblaeosque favos et hymettia foetu

Mella dari, dulcemque refert e cortice rorem

Sponte quidem at succos longe indignanter amaros:

Usque adeo coelique situs solaque abdita terrae

Et soles variant ipsi positusque locorum.

Vor Kurzem brach die Jugend Portugals ${ }^{27}$ auf vom westlichen Ozean und durchquerte unbekannte Orte und feindliche Meere, begegnete nie zuvor gesehenen Ländern und Ufern und drang kühn ein in den brennenden Sand Afrikas und in unbekannte Städte, die das Meer umschließt. Von hier gen Süden, mit Blick zum westlichen Himmel, den Sternen der gegenüberliegenden Hemisphäre folgend, stieß sie vor in den unbekannten Wendekreis des Steinbocks, erstaunt über die unbekannten Dinge wie auch die Schatten der Orte. Von dort fuhr sie in entgegengesetzter Richtung hinauf zu den Küsten des Prasso (Cabo Delgado),

26 Liliana Monti Sabia: Echi di scoperte geografiche in opere pontaniane. In: Columbeis V. Hg. von Stefano Pittaluga. Genua 1993, S. 283-303, jetzt in: Studi su Giovanni Pontano, a cura di Giuseppe Germano. Hg. von Liliana Monti Sabia, Salvatore Monti. Bd. 2. Messina 2010, S. 1135-1157.

27 Das Adjektiv Calletia zu pubes ist eine Prägung Pontanos auf das klassische Callaeca/Callaica (Plin. Nat., 3,28; 8,166; Mart. 4,39,7; 10,37,4; Sil. 3,353). 
und den indischen Ozean und die Stürme des Raptus ${ }^{28}$ überwindend, erreicht sie endlich des Indus Ströme voller Edelsteine und die stets duftenden Ufer voller Zitrusbäume und berichtet, dass aus diesen Früchte gewonnen werden, süß wie der Honig der hybläischen Berge und des Hymettos, und dass aus der Rinde ein süßer Likör tropft, der Saft dagegen widerlich sauer sei: so sehr variieren Sonnenstand und Standort, Klima und Boden.

Es existieren also zwei verschiedene Typen von Zitrusfrüchten: die süße Zitruspflanze, die in Italien nur im Königreich Neapel und in einer bestimmten Gegend vorkommt, und die saure Pflanze. $\mathrm{Zu}$ dieser Unterscheidung gibt Pontano zwei Begründungen: die erste, mythologischen Charakters, besagt, dass die aus dem Körper des Adonis geborene Pflanze einerseits, in ihrer Bitterkeit, den Schmerz der Venus über den Verlust ihres Geliebten erneuert, und andererseits, in ihrer Süße, die Freude der Liebe; die zweite, wissenschaftliche Erklärung verbindet die süße oder bittere Ausformung der Frucht mit verschiedenen sie bedingenden Faktoren wie Klima, Bodenbeschaffenheit, Sonnenstand und Standort.

Im zweiten Buch der De hortis Hesperidum (V. 218-230) erzählt Pontano, wie die Zitronen der Nymphe Amalphis als Hochzeitsgabe dargebracht wurden: Amor erinnert seine Mutter in Vers 236-245 daran, sich nach Italien zu begeben, wo sie von Hymeneus und Amalphis an den Küsten der Sirenen erwartet wird, das heißt an den Küsten Kampaniens und, insbesondere, der Küste von Sorrent. ${ }^{29}$ Die Nymphe Amalphis schmückt mit den ihr geschenkten Zitronen und Orangenblüten Altäre und Brautlager (II 264-268):

Hinc et stirpis honos, hinc et Chariteis Amalphis

munere limonum et nemorum redolentibus auris

ornavit thalamos felixque Hymenaeos et aras

pinxit flore novo sparsitque Atlantide fronde

et passim stratis laetata est alga metallis.

Daher kommt der Ruhm des Stammes, und von daher schmückte Amalphis die Chariten mit Zitronen und dem duftenden Hauch jener Wälder und färbte glücklich Hochzeitsgemächer und Altäre mit jener exotischen Blüte und bestreute sie mit Atlantes Blättern und hier und da erfreute sich die Meeresalge an jenem Gold, das alles bedeckte.

28 Ptolemäus (Geogr., 9,1; 4,7,12; 28) spricht von einem Kap Rapton, von einem Gipfel Rapta, und einem Fluss Raptos, heute jeweils identifiziert mit Kap Ras Tontwe, mit der Stadt Pangani und dem Panganifluss in Tanganjika.

29 Nach Pontanos Überzeugung herrschten die Sirenen über die urzeitlichen Küsten ganz Kampaniens, insbesondere über die Stadt Sorrent, die - in einer persönlichen freien Etymologie des Humanisten - von ihnen den Namen bezog: Surentum quasi surenetum, insofern sedes der Sirenen. Dazu Iacono (Anm. 20), S. 183-184; 207. 
Die Verse erklären wiederum in ätiologischer Funktion die Verbreitung des Zitronenanbaus entlang der Küste Amalfis. Eine Stadt, bekannt für die Heilquellen, das wohltuende Klima und eben die Fülle an Zitronen, wie auch Pietro Summonte, Schüler und Kurator der posthumen Ausgabe der Opera inedita des Pontano in einer Anmerkung zu Urania I 1015 (S. 3704) erinnert:

Amalphis urbs maritima in Picentinis, citriorum copia, fontibus atque aeris salubritate nobilis, clara e Adreae Apostolis sepulchro.

Amalfi, Seestadt im Gebiet der Picentini, ist edel durch die Fülle der Zitrusfrüchte, die Quellen und das heilsame Klima und berühmt für das Grabmal des Apostels.

Der Abschnitt greift wiederum eine Anspielung aus dem Gesang der Parzen auf, der das erste Buch abgeschlossen hatte: ein Zauber, welcher der dem Körper des Adonis entsprungenen Pflanze Ewigkeit und Unsterblichkeit verleiht. Die Parzen prophezeiten das Schicksal des nova arbos (I 570-579): der Baum der Zitronatzitrone würde Schmuck aristokratischer und fürstlicher Paläste sein, das Holz für die Brautbetten liefern, seine Blüten würden Bräute schmücken, ${ }^{30}$ seine Frucht wäre Festmählern bestimmt und als Monument und Symbol der Venus, der Göttin der Liebe, würde er all jene, die in seinem Schatten verweilen, verliebt machen:

Tum Parcae auspicio cecinerunt omnia laeto:

„Et fructu felix et flore et fronde recenti

Vive, arbor, supera et seclis labentia secla,

Hortorumque honor et nemorum ac geniale domorum

Delicium, tua vel reges umbracula captent,

Ipsaque continuis iuvenum celebrere choreis;

Te convivia, te thalami nuptaeque frequentent,

Semper ament, quicunque tua versantur in umbra,

Assiduum referant frondes ver, aemulus aurum

Foetus et argento niteat flos concolor albo.“

Also verkündeten die Parzen in froher Verheißung all das: „Und du, Baum, lebe reich an Früchten, Blüten und immer frischen Blättern, überdaure die Jahrhunderte, die vorüberziehen, und (sei) Ehre der Gärten und Gehölze und festliche Wonne der Häuser; auch die Könige werden deinen kleinen Schatten suchen und du wirst gefeiert werden von nicht

30 Dass die Zitronatzitrone den Bräuten bestimmt war, kann zurückgeführt werden auf die antike Herkunft der Frucht aus dem Garten der Hesperiden, der unter Junos Schutz stand, da sie Hochzeitsgabe der Göttin zum Anlass ihrer Vermählung mit Zeus war: vgl. Apd. Bibl., 2,5,11; Serv. Ad Aen., 4,484. 
endenden Tänzen der Jugend; auf dich wird man zurückgreifen für Festmähler und Brautgemächer; wer in deinem Schatten verweilt, wird unaufhörlich lieben, deine Blätter mögen dem ewigen Frühling nacheifern, deine Frucht sei Rivale des Goldes und deine Blüte strahle von gleicher Farbe wie weißes Silber.“

Die sich auf die Nymphe Amalphis beziehenden Verse eröffnen - gemäß einer präzisen diegetischen und strukturalen Praxis - einen Abschnitt mit ausführlichen Vorschriften zur Kultivierung der amalfitanischen Zitronen. Diesem geht aufgrund seiner Bedeutung eine Apostrophe an die Chariten voraus und an Nymphen, die dem klassischen Olymp unbekannt sind (De hortis Hesperidum, II 220-229):

Adsitis, nymphae Cyrenides, o mihi, nymphae

Masitholae, aspirate, o quae Paliurides umbra

Gaudetis: iuvat et fluvios habitare recessus.

Et citri iuvet umbra, iuvent limonides aurae,

Hesperidum et veteres cantu renovemus honores.

Seid mir wohlgesonnen, ihr kyrenischen und masitholischen Nymphen, schenkt mir Eingebung, oh ihr Paliuriden, die ihr Gefallen findet am Schatten: es erfreut auch, die verborgenen Winkel der Flüsse zu bewohnen. Und so mag der Schatten der Zitronatzitrone helfen, der duftende Hauch der Zitronen, und wir erneuern durch unseren Gesang die antike Ehre der Hesperiden.

Diese Zeilen zur Beschreibung der Herkunft der Zitronen an den Küsten Amalfis sind voller Anspielungen, zu deren Exegese es notwendig ist, ein wenig näher auf den gelehrten Hintergrund der Konstruktion einzugehen. ${ }^{31}$ Während sich bei den Cyrenides die direkte Verbindung zur antiken griechischen Kolonie Kyrene im heutigen Libyen recht problemlos herstellen lässt, muss man zur Identifikation der sogenannten Masitholae und Paliurides eine Ausgabe der Geographike Hyphegesis des Ptolemäus ${ }^{32}$ (insbesondere Geogr., 4,6,8) in den Händen

31 Siehe Antonietta Iacono: Territorio, poesia ed erudizione nel De hortis Hesperidum di Giovanni Pontano. In: Acta Conventus Neolatini Vindobonensis (Proceedings of the Sixteenth International Congress of Neo-Latin Studies - Vienna 2015). Hg. von Astrid Steiner, Franz Roemer. Leiden, Boston 2018, S. 358-368.

$32 \mathrm{Zu}$ Pontanos wissenschaftlichen und astronomischen Interessen siehe Michele Rinaldi: La lettera di dedica a Federico da Montefeltro del primo libro delle Commentationes in centum sententiis Ptolemaei di Giovanni Gioviano Pontano. In: Cahiers de Recherches Médiévales et Humanistes 25 (2013), S. 341-355; Pontano e le tradizioni astrologiche latine medievali: le postille dell'umanista al codice CLM 234 della Bayerische Staatsbibliothek di Monaco e nel Barberiniano latino 172 della Biblioteca Apostolica Vaticana. In: Atti Accademia Pontaniana 52 (2003), S. 295-324. 
haben. Demnach sind die Masitholae eine Transfiguration eines unbekannten libyschen Flusses, des Masitholos, während die Paliurides die Sümpfe in der Nähe von Kyrene darstellen, das wiederum in der Nähe des Gartens der Hesperiden verortet wurde. Die Verflechtung der Quellen dient zur Schaffung eines Aition, das besonders wichtig ist zur Einführung der monographischen Sektion, die den Zitronen von Amalfi gewidmet ist, denn diese werden von Pontano Masitholi genannt, d. h. Früchte antiken libyschen Ursprungs, die als Brautgabe der Venus an die Nereide Amalphis nach Amalfi gelangten.

Erst in den Versen 386-411 des ersten Buches wird schließlich der Körper des Adonis in seiner Unversehrtheit und ganzen Schönheit beschrieben. Adonis steht hier sinnbildhaft für die Schönheit, die Venus in ihren Zitrusgärten sehen will, denn diese - so betont der Dichter - sollen zugleich Spiegel und Bild der Schönheit des Geliebten Adonis sein:

\footnotetext{
Nunc, quae sit formae ratio et quae cura, docendum, Undique quo decor ipse sibi et nova gratia constet, Nec frustra veteres Veneris referentur amores. Non alias cultu maiore incessit Adonis Venatum, non Niliacas spectatior unquam In silvas, non ante Venus maiore paratu Ornarat, quam luce quidem, qua fossus ab apro Concidit et nigras tabe madefecit arenas.

Jetzt ist es Zeit zu lehren, welches das Kriterium und die Pflege der Schönheit sein sollte, wie überall Eleganz und ungewöhnliche Anmut sich bilden, und dass die alten Liebesgeschichten der Venus nicht umsonst erzählt werden. Ebenso begab sich Adonis mit größtem Aufzug zur Jagd, nie schöner, in die Gehölze des Nils, nie zuvor hatte Venus ihn prächtiger geschmückt als an jenem Tag, an dem er zerrissen vom Eber niedersank und die schwarze Erde mit seinem Blut tränkte. [...] So prunkvoll geschmückt sandte ihn die Göttin in die schattigen Wälder und mit den Zeichen ihrer Küsse auf seinen rosigen Lippen; also fordert die Göttin die Pflege jener Bäume, und dass sie in Pracht und Gold auf lange Zeit eben in diesen Gärten erstrahlen.

Die Darstellung des zur letzten Jagd gekleideten Adonis dient einem ausführlichen, didaktischen Abschnitt zur Kunst des Formschnitts, der eingefasst ist zwischen dieser poetischen Szene und dem Epilog des ersten Buches mit dem Gesang der Parzen. Die Schönheit des Adonis und seines Körpers werden als Kriterien zur Anlage der Zitrusgärten ausgewiesen, im Sinne einer eurythmia, der Idee von Anmut und Harmonie, die auf den idealisierten Maßverhältnissen 
des menschlichen Körpers gründet und in der humanistischen Renaissance weit verbreitet war. ${ }^{33}$ Die vollständige, detaillierte Beschreibung des jungen Liebhabers der Venus von Kopf bis Fuß entspricht ganz dem Regelsystem der artes poeticae. Mit malerischen Effekten überhöht sie die Figur des Adonis, und auch die verzögerte Darstellung des vollständigen Portraits des Jünglings durch den Dichter verstärkt ihre Bedeutung (De hortis Hesperidum, I 394-407):

\author{
Cingebat crinem myrrae de palmite ramus, \\ Ad frontem roseus diffulgebat hyacinthus, \\ Succincta et nitidum velabat purpura pectus, \\ Collaque fulgentes variabant candida baccae \\ Sparsim purpureis et coeruleis immistis, \\ Cingulaque auratis radiabant aspera bullis; \\ Elatis humeris pendebat aheneus ensis, \\ Ad capulum viridis fulgebat in orbe smaragdus, \\ Interstincta auro vagina, argentea cuspis, \\ Crura leoneae vestibant levia pelles, \\ Distinctique auro Tritones et aurea cymba, \\ Qua quondam paphias Venus ipsa enavit ad arces; \\ Horrebant manibus duro venabula cornu \\ Et paribus nodis paribusque nitentia gemmis.
}

Sein Haar schmückte ein vom Baum gepflückter Myrrhenzweig, auf der Stirn leuchtete eine scharlachrote Hyazinthe, ein kurzes purpurfarbenes Hemd bedeckte die reine Brust und auf seinem weißen Hals funkelten schillernd leuchtende Perlen und hier und da rote und himmelblaue Edelsteine; und am rauen Gürtel strahlte eine goldene Schnalle; von den hohen Schultern hing ein bronzenes Schwert, an dessen Griff leuchtete grün die Kugel eines Smaragdes, von Gold gezackt war die Scheide und von Silber die Spitze, die schlanken Beine waren in Löwenfell gekleidet und geziert mit goldenen Beschlägen von Tritonen und jenem Kahn, mit dem einst Venus selbst hinfuhr zum Felsen von Paphos; in seinen Händen hielt er aufrecht die Jagdspieße aus hartem Horn, gleichermaßen von Knoten und Edelsteinen strahlend.

Venus in Person hat ihren Liebhaber zu jener Jagd eingekleidet, die mit dessen Tod enden wird. Der Myrrhenzweig im Haar des Adonis evoziert seine Geburt aus einem Inzest und aus dem Leib einer Frau, die in einen Baumstamm verwandelt wurde (nach Ov. Met., 10,299-503), ist aber auch das Vorzeichen des kommenden Todes und der Metamorphose. Die Stirn schmückt eine scharlachrote Hyazinthe, eine zweideutige Anspielung, insofern sie die Metamorphose eines jungen Liebhabers des Apoll und seine Tötung durch den Gott

33 Zum Ideal der anthropozentrischen concinnitas siehe Rudolf Wittkower: Principi architettonici nell'età umanistica. Turin 1964. 
selbst erinnert (wiederum Ov. Met., 10,162-219), ${ }^{34}$ und andererseits ein rötlicher Edelstein ganz ähnlich dem Amethyst (Plin. Nat., 37,125). Dass die Hyazinthe als Edelstein $\mathrm{zu}$ interpretieren ist, der die Stirn des Adonis nach der Mode des fünfzehnten Jahrhunderts schmückt, bestätigt eine stimmungsvolle Passage in der Hypnerotomachia Poliphili, ${ }^{35}$ einem Werk, das Pontano meines Erachtens bekannt gewesen sein dürfte: hier ist zu lesen von einer petra pretiosa di Iacyntho, di colore vermiglio transparente cum grande corruscatione di flammeo splendore, die das Grabmal des Adonis schmückt. Brust und Hals des Adonis zieren eine purpurfarbene Stola, die seinen strahlenden Teint betont und eine Kette aus Perlen und Edelsteinen, ein Ornat, der eher einer adligen Dame zukommt als einem Jüngling. ${ }^{36}$ Die visuelle Dimension dieses Abschnitts scheint die effictio Adonidis auf ein weibliches Modell zu stimmen, die den Epheben in eine Art ,Double‘ der Venus verwandelt. Die prunkvolle magnificentia, mit der Venus Adonis auf die letzte Jagd vorbereitet, weist auf jene hin, die die Göttin für die Zitrusgärten fordert: im lebendigen, toten, vergöttlichten, in einen Baum verwandelten Adonis spiegeln sich Vorliebe und Genuss am Schönen sowie der Wille der Göttin. Diese magnificentia zeichnet auch Kleidung und Jagdwaffen des Jünglings aus: Adonis trägt ein typisches Jagdkleid, jedoch aus Löwenfell, weiter ausgeschmückt mit Schnallen in Gestalt von Tritonen und des Kahns von Paphos. Ein Gürtel mit einer goldenen Schnalle, ein Bronzeschwert mit Spitze aus Silber und einem Griff, den ein Smaragd ziert, eine Schwertscheide aus Kettengold, Jagdspieße aus

34 Pontano verwendet die Hyazinthe in seiner Dichtung mehrfach in Verbindung mit Tod und Trauer, zum Beispiel in Tum., II 24: Tumulus Ariadnae Saxonae Neapolitanae. Viator, Genius et Hyacinthus colloquuntur, zum Tod seiner Ehefrau Adriana Sassone, auch in Lyra, VIII 19. Zur Symbolik der Botanik bei Pontano siehe Carmela Vera Tufano: Lingue tecniche e retorica dei generi letterari nelle Eclogae di G. Pontano. Neapel 2015, passim; Hélène Casanova-Robin: Des métamorphoses végétales dans les poésies de Pontano: Mirabilia et lieux de mémoire. In: La mythologie classique dans la littérature néo-latine. Hg. von Virginie Leroux. Clermont-Ferrand 2011, S. 247-268; Dies.: Dendrophories d'Ovide à Pontano: la nécessité de l'hypotypose. In: Ovide. Figures de l'hybride. Illustrations littéraires et figurées de l'esthétique ovidienne à travers les âges. Hg. von Hèléne Casanova Robin. Paris 2009, S. 103-124.

35 Francesco Colonna: Hypnerotomachia Poliphili. Hg. von Giovanni Pozzi, Lucia A. Ciapponi. Padua, Antenore 1980, S. $386=$ z6v.

36 Die vornehme Portraitkunst des fünfzehnten Jahrhunderts bietet zahlreiche Vergleichsmodelle: Maria Giuseppina Muzzarelli: Ma cosa avevano in testa? Copricapi femminili proibiti e consentiti fra Medioevo ed Età moderna. In: Un bazar di storie. A Giuseppe Olmi per il sessantesimo Genetliaco. Hg. von Claudia Pancino, Renato G. Mazzolini. Trient 2006, S. 13-28; Dora Liscia Bemporad: Il gioiello al tempo di Piero. In: Con gli occhi di Piero. Abiti e gioielli nelle opere di Piero della Francesco. Catalogo della mostra (Arezzo, basilica inferiore di San Francesco 11 luglio-31 ottobre 1992). Hg. von Maria Grazia Ciardi Duprè, Giuliana Chesne, Dauphiné Griffo. Venedig 1992, S. 81-87. 
Horn, von Edelsteinen geziert, vervollständigen die Armatur. Die visuelle Komponente, verstärkt durch das Nebeneinander und den Wechsel der Farben, ${ }^{37}$ steigert die Anschaulichkeit und bildliche Potenz dieser Verse - diese Ekphrase ist eines der schönsten Beispiele der enargheia Pontanos und der humanistischen Dichtung allgemein. ${ }^{38}$ Pontano erhöht die Adonis-Figur hiermit zum Modell jener magnificentia, die die repräsentativste Chiffre des neapolitanischen Hofes war und allgemein der aristokratischen Identität des edlen Fürsten und des Edelmannes, ${ }^{39}$ wobei die Stilisierung gleichermaßen zur literarischen wie malerischen Rezeption einlädt.

Diese drei Beispiele können leider nur eine vage Idee geben von der kühnen Konstruktion, die Pontano mit De hortis Hesperidum realisiert hat. Alle Ursprungsmythen haben zugleich ätiologischen wie funktionalen Charakter: die ersten beiden dienen dazu, einzelne Abschnitte einzuführen, wie die Kultivierung der Zitronen von Amalfi und die Kunst des Formschnitts angewandt auf Zitrusgärten; das dritte Aition dient der Unterscheidung zwischen süßen und sauren Zitrusfrüchten, denen der Dichter einen ausführlichen Abschnitt im zweiten Buch widmet.

37 Siehe Antonietta Iacono: Nitidum velabat purpura pectus. La vestizione di Adone nel De hortis Hesperidum di Pontano. In: L'esegeta appassionato. Studi in onore di Crescenzo Formicola. Hg. von Mario Lentano, Olga Cirillo. Mailand, Udine 2019, S. 139-151.

38 Gemäß den klassischen Rhetorikregeln (vgl. im Besonderen Quintilians Inst., 8,3,61-71) verwirklichen die Verse Pontanos das Ideal der Dichtung als sprechender Malerei, wie sie die humanistische Poetik theorisiert, vgl. Marcello Ciccuto: Spirantia signa. Cultura ecfrastica di Agnolo Poliziano, In: Ecfrasi: Modelli ed esempi tra Medioevo e Rinascimento. Hg. von Gianni Ventura, Monica Farnetti. Rom 2004, S. 123-138.

39 Siehe Amedeo Quondam: Forma del vivere. L'etica del gentiluomo e i moralisti italiani. Bologna 2010, S. 249-431; Matthias Roick: Pontano's virtues. Aristotelian moral and political Thought in the Renaissance. London 2017. 